\title{
Récurrence ipsilatérale de la neuropathie optique ischémique antérieure non artéritique : Rapport de cas
}

\author{
Daniel Brigham, OD, FAAO \\ Carl Vinson Veterans Affairs \\ Medical Center
}

\begin{abstract}
Résumé
La neuropathie optique ischémique antérieure non artéritique (NOIA-NA) est une maladie multifactorielle qui constitue le type le plus courant de neuropathie optique ischémique. La NOIA-NA apparait dans un œil et peut se développer plus tard dans l'autre œil. La récurrence ipsilatérale de la NOIA-NA est rare et plusieurs théories ont été proposées pour l'expliquer. Le présent rapport de cas décrit un patient chez qui une récurrence unilatérale de la NOIA-NA est survenue, examine les étiologies proposées et met l'accent sur les symptômes visuels à la suite de sa résolution.
\end{abstract}

\section{KEY WORDS:}

œdème du nerf optique, neuropathie optique ischémique non artéritique récurrente, sensibilité au contraste, couche des fibres nerveuses rétiniennes péripapillaires (CFNRp)

\section{INTRODUCTION}

La neuropathie optique ischémique antérieure non artéritique (NOIA-NA) représente $95 \%$ de toutes les neuropathies optiques ischémiques antérieures ${ }^{1}$. C'est l'une des maladies les plus visuellement débilitantes chez les patients de plus de 50 ans, avec une incidence annuelle estimée aux États-Unis de 2,3 à 10,2 pour 100000 habitants, ce qui équivaut à environ 6000 nouveaux cas par an ${ }^{1}$. La NOIA-NA touche une proportion nettement plus élevée de Caucasiens que d'autres races, avec un âge moyen d'apparition de 66 ans ${ }^{1}$. L'acuité visuelle au moment de la présentation initiale est variable ; $49 \%$ des patients ont une acuité visuelle initiale de $\geq 20 / 64$, tandis que de $23 \%$ à $34 \%$ ont une acuité visuelle initiale de $\leq 20 / 200^{1,2}$. De plus, $38 \%$ ont des défauts du champ visuel minimes à légers et $43 \%$ ont des défauts marqués à graves ${ }^{2}$.

La pathogenèse de la NOIA-NA est controversée, mais deux mécanismes ont été proposés pour la décrire. Le mécanisme le plus communément accepté est l'ischémie transitoire ou l'hypoperfusion de la tête du nerf optique $(\mathrm{TNO})^{3}$. La deuxième cause proposée est l'occlusion embolique des artérioles qui alimentent la $\mathrm{TNO}^{3}$. En ce qui concerne la première, il existe deux grandes catégories de facteurs de risque : les facteurs de risque prédisposants (différenciés en facteurs de risque systémiques et facteurs de risque locaux de la TNO) et les facteurs de risque précipitants ${ }^{4}$.

L'apparition de la NOIA-NA dans l'œil contralatéral est possible, et survient chez environ $25 \%$ des patients dans les trois ans suivant son apparition dans l'œil primaire ${ }^{5}$. Toutefois, la récurrence dans le même œil est rare. Dans une étude portant sur 594 patients consécutifs atteints de NOIA-NA, seulement $6 \%$ ont développé une NOIA-NA une deuxième fois dans l'œil ipsilatéal ${ }^{6}$. Le présent rapport de cas suivant met en évidence une récurrence ipsilatérale de NOIA-NA et approfondit les étiologies proposées de la NOIA-NA, en mettant l'accent sur la récurrence ipsilatérale. De plus, on y discute des problèmes visuels courants associés à la NOIA-NA. 


\section{RAPPORT DE CAS}

En 2017, un homme caucasien de 76 ans s'est présenté à notre clinique pour se plaindre de l'apparition récente de taches brunes intermittentes de formes et de tailles différentes dans le champ de vision central de son œil droit. Ces taches avaient commencé trois jours auparavant et apparaissaient quelques fois par jour. De plus, il a décrit une « lueur » sur le côté droit de son champ de vision associée à une douleur sourde derrière son œil droit. Il a affirmé ne pas avoir de maux de tête, de sensibilité au cuir chevelu, de claudication de la mâchoire, de fièvre, de perte de poids, de phénomène d'Uhthoff, d'engourdissement ou de faiblesse.

Ses antécédents médicaux ont révélé qu'il souffre d'hypertension, d'hyperlipidémie, de coronaropathie, d'insuffisance veineuse, de reflux gastro-œsophagien, d'insomnie et qu'il a souffert d'une paralysie de Bell du côté gauche 20 ans auparavant. Les médicaments actuels comprenaient le citalopram (Celexa), le furosémide (Lasix), le lisinopril (Zestril), le métoprolol (Lopressor), la nitroglycérine (Nitrostat), l'oméprazole (Prilosec), la simvastatine (Zocor) et $325 \mathrm{mg}$ d'aspirine (Bayer). Il a nié l'utilisation d'inhibiteurs de phosphodiestérase-5, de tabac, d'alcool ou de drogues récréatives.

Ses antécédents oculaires comprenaient une NOIA-NA OS en 2007 et des cataractes visuellement insignifiantes OU. Il a affirmé n'avoir aucun antécédent de chirurgie oculaire, de traumatisme oculaire et aucun antécédent familial de maladie oculaire.

Sa tension artérielle était de 142/79 mm Hg à 14 h 30.

Sa meilleure acuité visuelle corrigée était de $20 / 20^{-1}$ OD et le comptage des doigts à 0,3 mètre OS. Il avait une mobilité oculaire normale sans douleur ni vision double. Les pupilles étaient égales et rondes avec un défaut pupillaire relatif de $3^{+}$à gauche. La périmétrie par confrontation a révélé une restriction du côté nasal inférieur OD et une restriction inférieure complète OS. Les tests de vision chromatique au moyen de plaques pseudo-chromatiques HRR ont révélé une vision intacte OD (6/6 plaques) et ne pouvaient pas être effectués OS en raison de son acuité profondément réduite. Les artères temporales superficielles de droite et de gauche étaient facilement palpables et non sensibles. La biomicroscopie à la lampe à fente n'a rien montré de particulier, à l'exception de cataractes sclérotiques nucléaires de grade $2^{+}$OU. Les pressions intraoculaires étaient de $15 \mathrm{~mm} \mathrm{Hg} \mathrm{OU}$ à 14 h, mesurées à l'aide de la tonométrie d'applanation de Goldmann.

L’examen du fond de l'œil dilaté a révélé un œdème de la région supérieure de la TNO OD avec une hémorragie unique, quoiqu'importante, de la couche de fibres nerveuses rétiniennes à la marge nasale (figure 1). La TNO gauche montrait une pâleur diffuse avec des marges distinctes (Figure 2). À titre de référence, on avait précédemment déterminé que les rapports excavation-papille étaient de 0,3 (rond) OD et de 0,25 (rond) OS avec un disque optique de 1,6 mm OU. Le reste de l'examen du fond de l'œil n'a rien montré de particulier.

Figure 1 : Photographie du fond de l'œil (OD) prise lors de la visite initiale. On peut clairement distinguer l'œè̀me de la région supérieure de la TNO avec une hémorragie en forme de flamme du côté nasal.

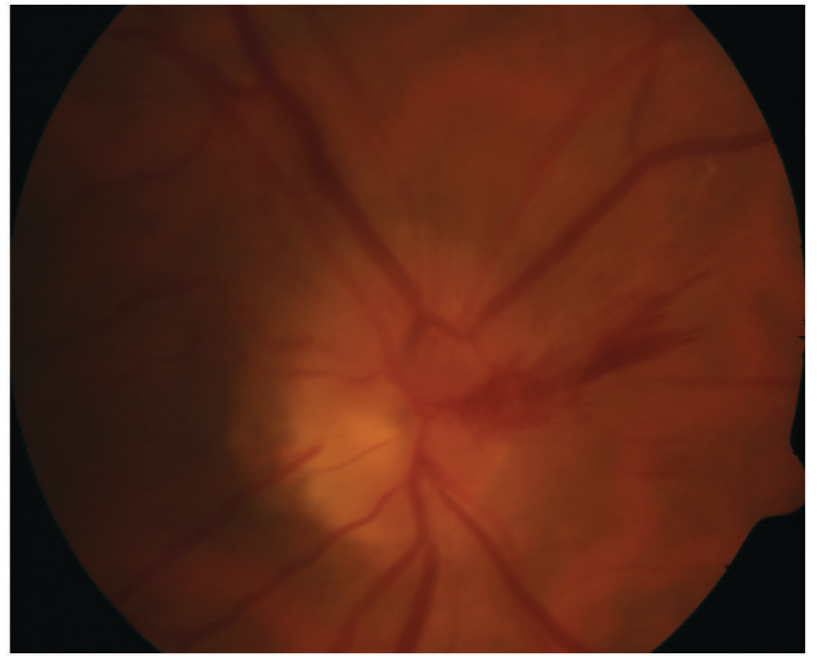


Figure 2 : Photographie du fond de l'œil OS montrant la pâleur diffuse de la TNO.

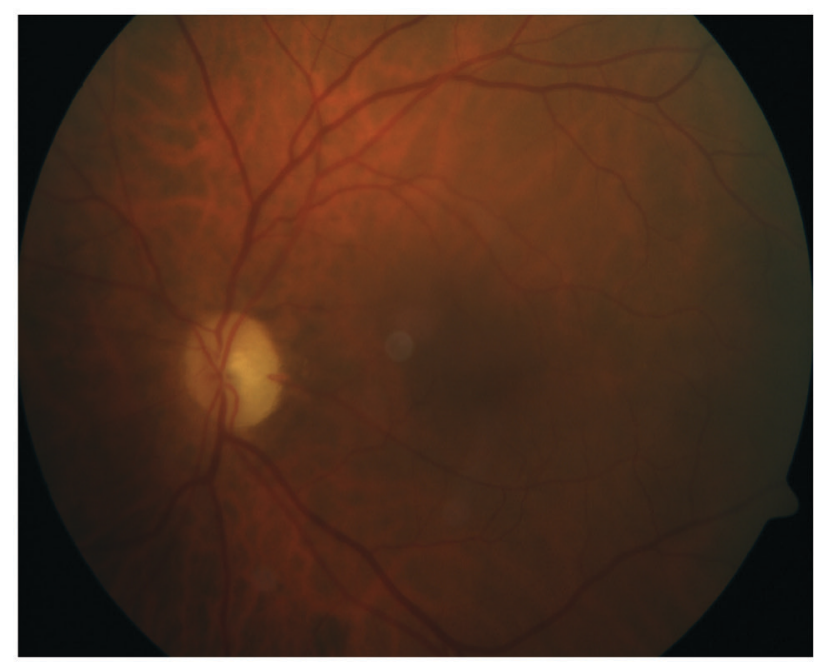

D'autres analyses ont été effectuées, notamment l'imagerie par tomographie à cohérence optique en spectral domaine (TCO-DS) de la couche de fibres nerveuses rétiniennes péripapillaires (CFNRp) (figure 3). Ce résultat a été obtenu avec un appareil TCO-DS Spectralis de Heidelberg (Heidelberg Engineering, Inc., Heidelberg, Allemagne) et a révélé un œdème de la CFNRp dans l'hémisphère supérieur et une épaisseur normale en inférieur.

Figure 3 : La TCO-CFNR OD acquise lors de la visite initiale montre un épaississement dans l'hémisphère supérieur.

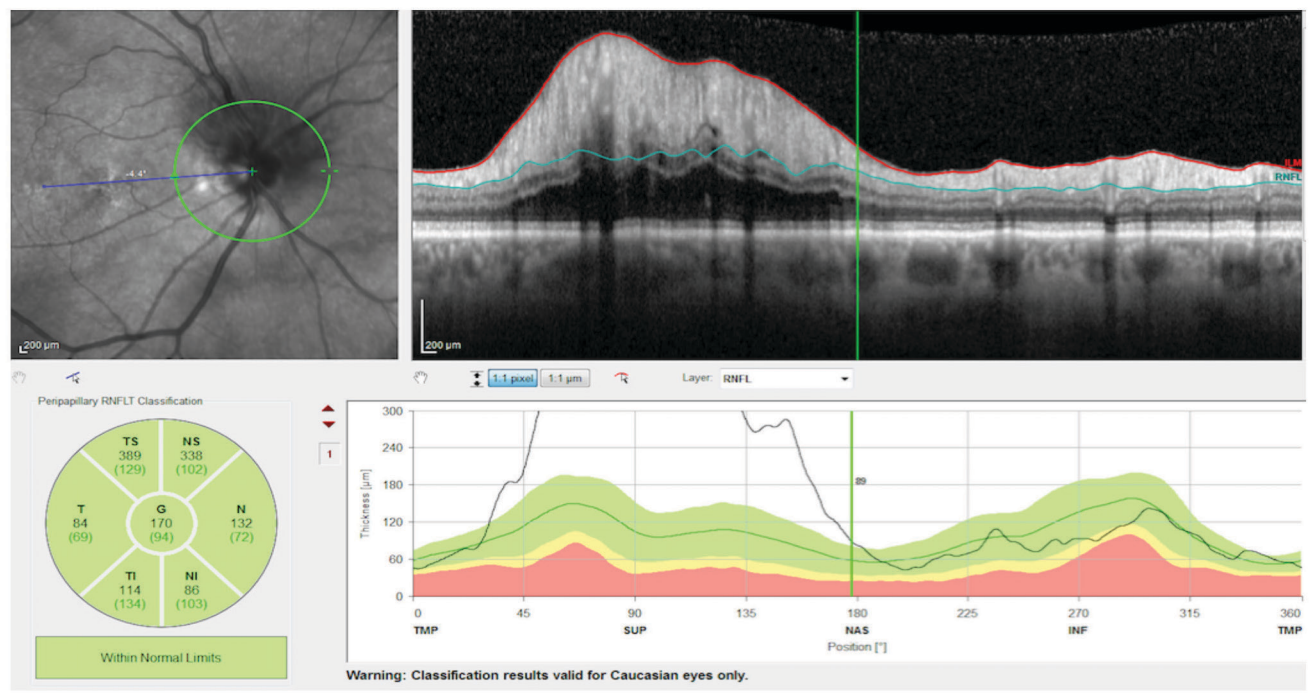

Les diagnostics différentiels envisagés pour ce patient comprenaient d'autres causes possibles de l'œdème de la TNO, ainsi que des causes possibles de l'œdème de la TNO dans un œil et de la pâleur de la TNO dans l'autre œil. Parmi celles-ci, mentionnons la démyélinisation (sclérose en plaques), l'inflammation (sarcoïdose, lupus érythémateux systémique), la compression (méningiome du nerf optique, syndrome de Foster Kennedy), l'infection (tuberculose, bartonella, syphilis, maladie de Lyme), une néoplasie (lymphome, leucémie, gliome) et des étiologies vasculaires (neuropathie optique ischémique antérieure artéritique et non artéritique (NOIA-A, NOIA-NA) ${ }^{7}$. 
Malgré le diagnostic présomptif de NOIA-NA, des tests en laboratoire ont été effectués le même jour et comprenaient un profil métabolique complet (CMP), une formule sanguine complète, la vitesse de sédimentation (VS), la protéine C-réactive (CRP) et une hémoglobine glyquée (HBAlc). Tous les résultats se situaient dans la plage de référence normale pour l'âge du patient et les comorbidités existantes; plus précisément, une VS de $7 \mathrm{~mm} / \mathrm{h}$ et une CPR de moins de 5,0 mg/L ont aidé à exclure une NOIA-A secondaire à la maladie de Horton. Une étude antérieure sur le sommeil n'a révélé aucune apnée du sommeil.

En raison de la présentation clinique et des résultats normaux des tests de laboratoire, un diagnostic de NOIA-NA a été posé. Le patient a été informé des résultats et de l'évolution de la maladie, ainsi que de la façon de mieux contrôler les facteurs de risque potentiels de la NOIA-NA, y compris ses maladies systémiques sous-jacentes. De plus, le moment de l'utilisation de ses médicaments antihypertenseurs systémiques a été discuté en détail. Le patient a affirmé ne pas utiliser ses médicaments antihypertenseurs systémiques dans les 3 à 4 heures précédant son endormissement malgré la modification des habitudes de sommeil en raison de l'insomnie. Une note a été envoyée à son fournisseur de soins primaires avec les résultats des examens de la journée. On a demandé au patient de retourner à la clinique une semaine plus tard pour recevoir des soins continus, y compris un examen du fond de l'œil dilaté, une TCO-DS et un examen du champ visuel Humphrey (HVF 24-2).

Une semaine plus tard, le patient s'est présenté sans nouvelle plainte visuelle ou systémique. Sa meilleure acuité visuelle corrigée était de $20 / 20^{-2}$ OD et le comptage des doigts à 0,3 mètre OS. Les résultats des autres tests préliminaires étaient stables et semblables à ceux de la visite précédente. L'examen du fond d'œil dilaté montrait que la TNO OD avait la même apparence (figure 4), bien que la TCO-DS ait révélé une légère diminution de l'épaisseur de la CFNRp supérieure correspondant à la résolution de l'œè̀me OD (figure 5). Il n'a pas été possible d'effectuer un examen HVF 24-2 lors de cette visite en raison d'une défectuosité de l'équipement. Le patient a reçu une formation sur l'évolution de la NOIA-NA et on lui a recommandé de revenir un mois plus tard pour subir le HVF 24-2.

Figure 4 : Photographie du fond de l'œil (OD) une semaine après la présentation initiale.

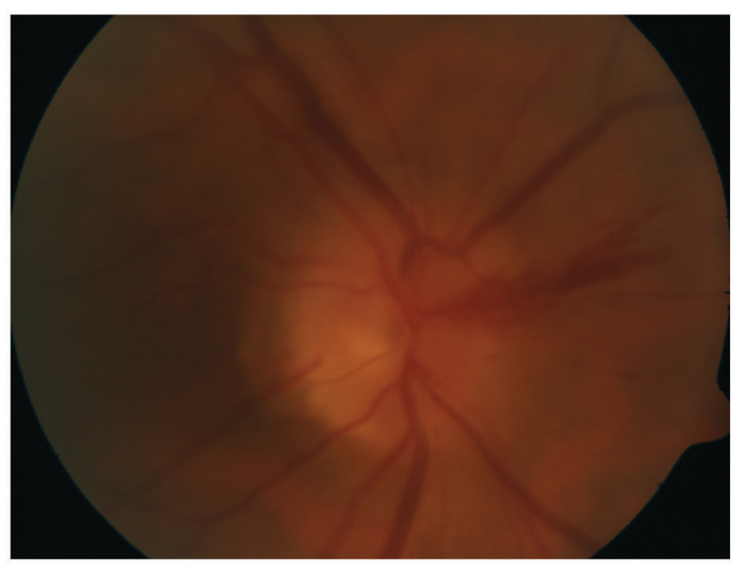

Au cours de la visite de suivi de la 10e semaine (le patient a manqué son suivi de la cinquième semaine), le patient s'est à nouveau plaint d'une diminution de l'acuité visuelle centrale dans l'œil droit pendant trois jours. De plus, il a remarqué des changements dans sa capacité de distinguer les nuances et a constaté une augmentation de la sensibilité à la lumière. Sa meilleure acuité visuelle corrigée avait baissé et était de 20/30- OD. Le test de sensibilité au contraste Mars Letter de l'œil droit a révélé une perte de sensibilité au contraste grave, avec une valeur logarithmique de 0,56.

L'examen du fond de l'œil dilaté a démontré une résolution quasi complète de l'œè̀me de la région supérieure de la TNO et de l'hémorragie de la CFNR, mais un œè̀me de la région inférieure de la TNO était maintenant visible (figure 6). La TCO-DS a confirmé cette présentation en révélant l'amincissement de la CFNRp dans le secteur temporal supérieur et son épaississement dans l'hémisphère inférieur (figure 7). Un examen HVF 24-2 utilisant une stratégie standard SITA OD était fiable avec des pertes de fixation de 1/18, $0 \%$ de faux positifs, $20 \%$ de faux négatifs et une excellente poursuite oculaire. L'écart moyen était de $-16,50 \mathrm{~dB}$ et l'indice de champ visuel était de $46 \%$. Il y avait des défauts centraux inférieurs et supérieurs denses qui correspondaient à l'atteinte de la TNO supérieure antérieure et à celle de la TNO inférieure actuelle, respectivement (figure 8). L'examen HVF 24-2 de l'œil gauche n'a pas été tenté en raison d'une perte de vision profonde. 
Figure 5 : La TCO-CFNR OD acquise une semaine après la présentation initiale montre une amélioration de l'épaississement dans l'hémisphère supérieur et un léger épaississement du côté nasal.

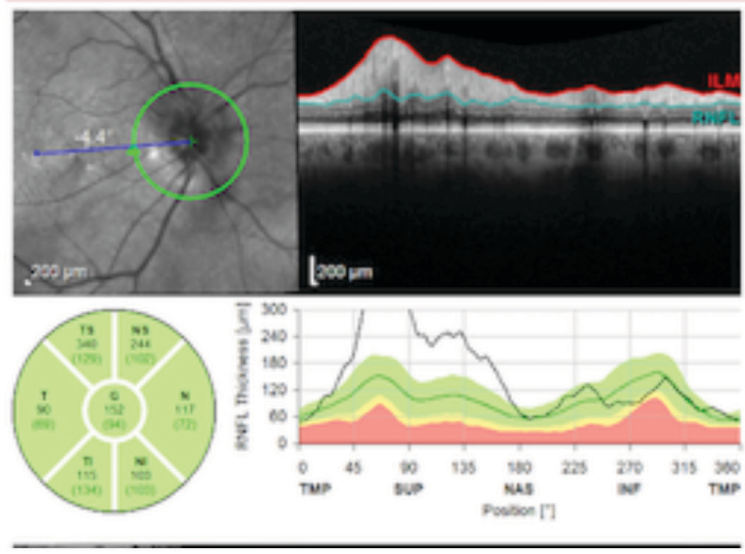

\section{Follow-Up $=1$ Mar/2/2017}

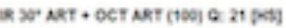

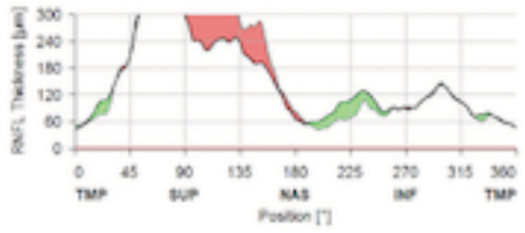

Classification

Within Normal Limits

Figure 6 : La photographie du fond de l'œil $(O D)$ prise six semaines après la présentation initiale montre maintenant un œdème dans la région inférieure de la TNO.

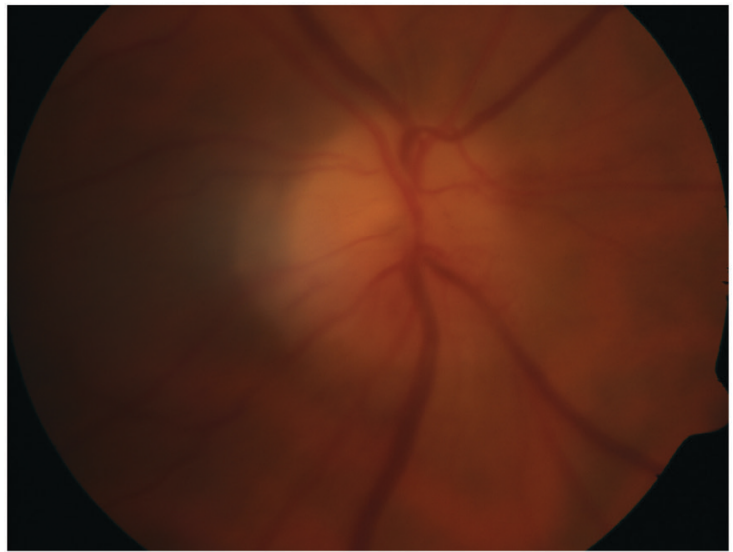

Figure7 : La TCO-CFNR OD acquise six semaines après la présentation initiale montre une résolution complète de l'épaississement dans l'hémisphère supérieur, un amincissement dans le secteur temporal supérieur et un nouvel épaississement dans l'hémisphère inférieur.
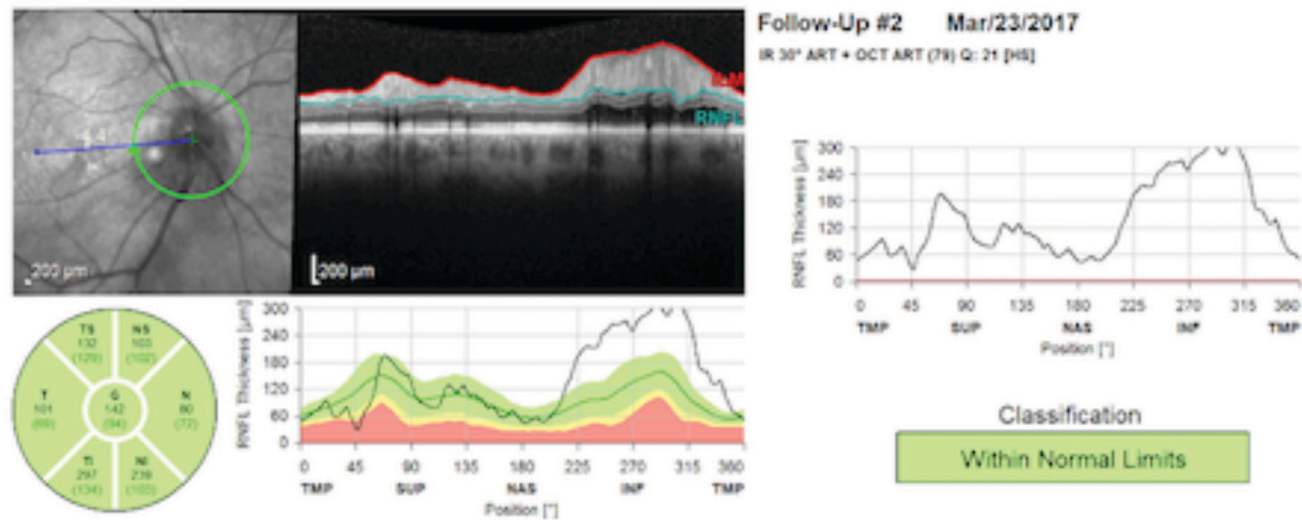
Figure 8 : Examen 24-2 standard Sita du champ visuel de Humphrey après la présentation initiale et la NOIA-NA OD récurrente.

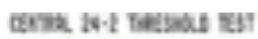

frata somen catio ont

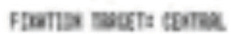

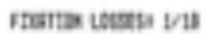
fas F⿱ rase st tanci an nets vestis: tors

inst or

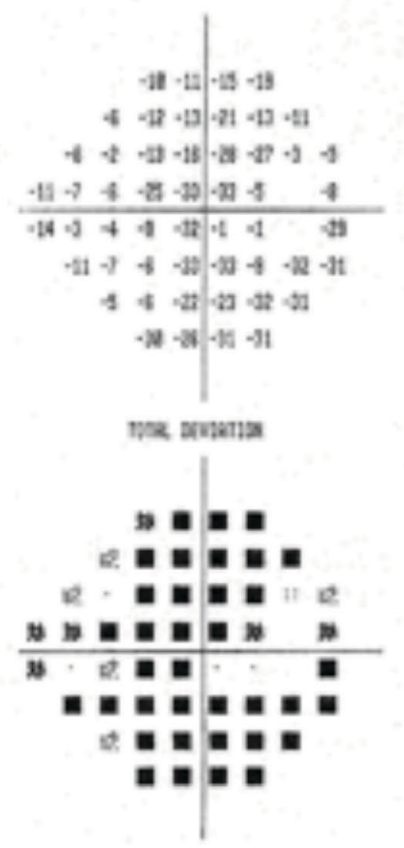

รtous: Ets with

woxsoust Ha wa

starts the tracen nat netw: 2.10

vaka maim

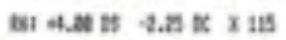

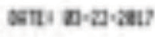
TE: this is atri $n$ 
Le patient a été informé de la rareté de la récurrence ipsilatérale de la NOIA-NA. L'importance de contrôler les facteurs de risque systémiques potentiels a été renforcée, ce qui a amené le patient à admettre qu'il ne prenait pas ses médicaments d'ordonnance correctement. De plus, il a mentionné que son horaire de sommeil avait changé au cours des dernières semaines en raison du stress, et qu'il avait commencé à prendre ses médicaments antihypertenseurs systémiques plus près de l'heure du coucher.

Le patient devait revenir après un mois pour un examen du fond de l'œil dilaté avec TCO-DS OD et pour refaire le HVF 24-2 OD. Il a également été aiguillé vers des services de réadaptation de la vue pour l'aider à améliorer les activités quotidiennes qui avaient été touchées.

Un mois plus tard (suivi de la 14e semaine), l'œdème de la TNO était complètement résolu. Le disque montrait alors une pâleur diffuse, secondaire au processus atrophique qui avait suivi l'œè̀me (figure 9). Le défaut du champ visuel et l'acuité visuelle sont demeurés inchangés. Le patient avait rendez-vous pour une évaluation en vue de la réadaptation de la vision et devait revenir quatre mois plus tard pour passer un HVF 10-2. Le patient n'est pas revenu pour des examens de suivi au-delà de ses soins de réadaptation visuelle.

Figure 9 : La photographie du fond de l'œil (OD) prise dix semaines après la présentation initiale et quatre semaines après les récidives ipsilatérales montre une réduction de l'œè̀me dans la région inférieure de la TNO et une augmentation de la pâleur de la TNO.

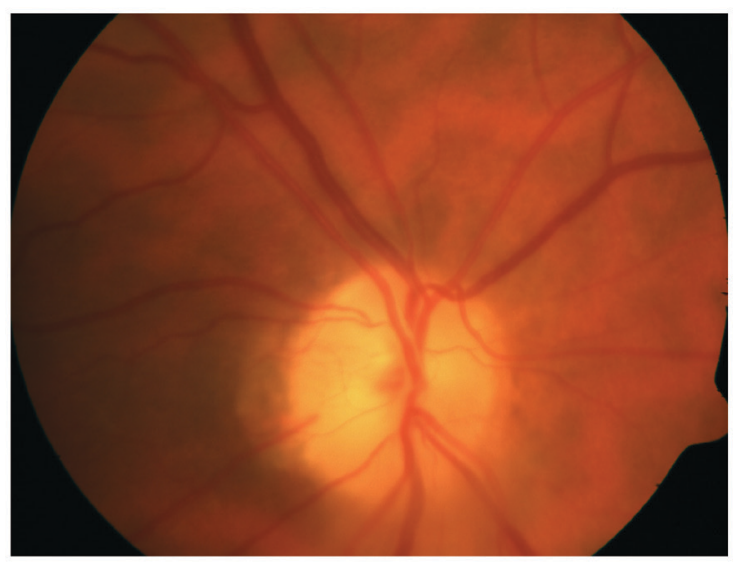

Au cours de l'examen de réadaptation visuelle, notre patient a démontré un problème de sensibilité au contraste et de photophobie que nous avons pu améliorer avec l'utilisation d'un filtre de couleur ambre claire à l'intérieur et d'un filtre de couleur ambre foncé sur monture enveloppante pour l'extérieur. On a également fourni au patient une loupe vidéo portative à contraste amélioré pour faciliter les tâches de lecture. Il n'était pas nécessaire de discuter des restrictions de conduite puisque le patient a cessé de conduire de son propre chef après sa première NOIA-NA.

\section{DISCUSSION}

La neuropathie optique ischémique antérieure non artérielle (NOIA-NA) est un trouble ischémique de la circulation de l'artère ciliaire postérieure (ACP), la principale source d'approvisionnement en sang de la tête du nerf optique (TNO) ${ }^{8-10}$. Des études plus récentes utilisant l'angiographie par tomographie en cohérence optique (A-TCO) ont montré une altération du flux dans les capillaires péripapillaires rétiniens (CPR) et les choriocapillaires péripapillaires (CCP) à la suite d'un épisode de NA-AION ${ }^{11,12}$. Ces domaines montrent une forte correspondance avec les changements fonctionnels et structurels $^{11}$. On peut supposer qu'une hypoperfusion des CPR après un épisode de NOIA-NA résulte d'un événement ischémique déclencheur dans la circulation de l'ACP ${ }^{11}$.Une des explications proposées pour cette diminution de la perfusion des CPR est que les CPR possèdent des caractéristiques anatomiques uniques que l'on ne trouve pas dans d'autres capillaires : elles ont tendance à être plus longues et plus droites, et relient directement les artères de gros calibre au long tronc veineux, sans anastomose ${ }^{12}$. Ces particularités rendent les CPR plus sensibles aux changements du flux sanguin, comme ceux qui se produisent lors d'événements ischémiques dans la circulation de l'ACP ${ }^{12}$. 
La pathogenèse de la NOIA-NA demeure controversée, mais deux mécanismes ont été proposés pour la décrire. La plupart du temps, une ischémie ou une hypoperfusion transitoire de la TNO est invoquée. À l'occasion, la NOIA-NA est attribuée à des lésions emboliques des artérioles alimentant la $\mathrm{TNO}^{8}$. Le premier mécanisme est décrit ci-dessous.

L’angiographie à la fluorescéine (FA) réalisée au début de la NOIA-NA a montré un remplissage retardé de la choroïde péripapillaire et de ses zones de bassin (une frontière entre les territoires de distribution de deux artères terminales quelconques) et non une absence totale de remplissage ${ }^{13}$. Cela peut être interprété comme signifiant que ces vaisseaux ne sont pas complètement bouchés ${ }^{13}$. Comme il n'y a qu'une perte transitoire de flux sanguin vers la TNO, la cascade ischémique et les dommages à la TNO qui en résultent sont généralement beaucoup moins graves. Cela a été démontré dans deux grandes études, dans lesquelles on a observé une amélioration visuelle spontanée dans environ $41 \%$ des yeux atteints d'une NOIA ${ }^{1,2}$. On suppose que cette hypoperfusion est le résultat de la chute de la pression de perfusion des capillaires de la TNO en dessous de la plage critique d'autorégulation ${ }^{8}$. L'autorégulation est essentielle pour maintenir un flux sanguin adéquat vers un tissu par la dilatation et la constriction des vaisseaux sanguins en réponse à la pression de perfusion et à la demande métabolique locale. Toutefois, l'autorégulation ne fonctionnera que sur une certaine plage de pression de perfusion et deviendra inefficace au-delà de cette plage. À ce stade, la circulation sanguine vers la TNO devient en corrélation directe avec la pression de perfusion ${ }^{14}$.

Plusieurs variables, dans différentes combinaisons, peuvent rendre la TNO plus vulnérable à une insulte ischémique. La combinaison particulière de facteurs qui donne lieu à la NOIA-NA sera unique à chaque patient touché, ce qui souligne la notion selon laquelle la NOIA-NA est de nature multifactorielle. ${ }^{8}$. Ces facteurs de risque peuvent être classés comme des facteurs de risque prédisposants. (différenciés en facteurs de risque systémiques et en facteurs de risque locaux propres à la TNO) ou des facteurs de risques précipitants ${ }^{4}$.

Les facteurs de risque prédisposants rendent la TNO plus sensible à un événement ischémique. Les facteurs systémiques comprennent l'hypertension artérielle, le diabète sucré, la cardiopathie ischémique, l'apnée du sommeil et l'hyperlipidémie, entre autres ${ }^{15-17}$. Plusieurs de ces facteurs de risque étaient présents chez notre patient. Les facteurs locaux propres à la TNO peuvent également jouer un rôle prédisposant, et comprennent l'absence de la cupule ou la présence d'une petite cupule dans le disque optique (facteur contributif secondaire) ${ }^{18}$, une PIO significativement élevée ${ }^{19}$, la présence de drusen dans le disque optique ${ }^{19}$, et l'emplacement du bassin versant formé par la distribution des artères ciliaires postérieures par rapport à la $\mathrm{TNO}^{20}$.

Le facteur de risque précipitant le plus notable, qui peut finalement constituer l'insulte finale au nerf sensible et initier la cascade d'ischémie qui prive la TNO, est l'hypotension artérielle nocturne ${ }^{21-23}$. Il a été montré que $73 \%$ des patients atteints d'une NOIA-NA ont spécifiquement déclaré que leur perte de vision avait été constatée au réveil après le sommeil ou une sieste ou lors de la première utilisation critique de leur vue ${ }^{24}$. En outre, la surveillance ambulatoire de la pression artérielle sur 24 heures a révélé de nombreux cas d'hypotension artérielle nocturne chez des personnes ayant développé une NOIA-NA ${ }^{22,23}$. Une relation significative entre l'hypotension artérielle nocturne et l'utilisation d'un traitement antihypertenseur oral puissant a été établie ${ }^{22,23}$. En fait, depuis l'avènement de ces agents de traitement hypotenseurs, on a constaté une augmentation de l'incidence de la NOIA-NA ${ }^{8}$. Dans ce cas, le patient prenait trois puissants médicaments hypotenseurs, et il a admis les avoir pris à l'approche du coucher avant la récidive ipsilatérale.

Chez les patients atteints de NOIA-NA, le risque d'atteinte de l'œil contralatéral varie de $14,7 \%$ à $25 \%$ sur une période de trois à cinq ans $\mathbf{s}^{5,25}$. Toutefois, la récurrence dans l'œil ipsilatéral est considérée comme rare. Il est important faire la distinction entre la véritable récurrence ipsilatérale et la progression de la NOIA-NA au stade aigu de la maladie.

L’œdème du nerf optique présente un schéma évolutionnaire caractéristique dans la NOIA-NA ${ }^{26}$. Au départ, une partie de la TNO présente un œedème, et le reste du disque n'est pas touché. Après plusieurs jours, un œè̀me généralisé et diffus peut se manifester sur la TNO tout entière. De façon séquentielle, la région de la TNO initialement atteinte développera une pâleur en réponse à l'insulte ischémique antérieure des fibres nerveuses. L'œdème peut persister dans le reste de la TNO. Cet œè̀me se résorbera aussi graduellement, avec la pâleur qui en résultera.

Dans ce cas, de la présentation initiale à une semaine après la présentation, il y a eu une certaine résolution de l'œdème supérieur et une légère augmentation de l'œdème du côté nasal inférieur (Figure 3 et Figure 5). Cette migration de l'œè̀me de son point de départ vers une zone non touchée à l'origine n'est pas rare d'après les constatations décrites précédemment. De la semaine 1 à la semaine dix après la présentation, l'œè̀me supérieur s'est entièrement résorbé, laissant un certain amincissement de la CFNRp. Cependant, un œdème inférieur beaucoup 
plus important que ce qui avait été observé lors de la migration initiale de l'œè̀me au cours de la présentation à une semaine s'était développé (Figure 5 et Figure 7). L'apparition d'un nouvel œedème important dans la région inférieure de la TNO, combinée au temps moyen médian de 7,9 semaines nécessaire à la résolution complète de l'œè̀me de la TNO après la NOIA-NA aigue ${ }^{26}$, confirme que le patient souffrait d'une NOIA-NA ipsilatérale récurrente.

Plusieurs hypothèses ont été avancées pour expliquer la rareté des récidives ipsilatérales de NOIA-NA, notamment l'incapacité du patient à reconnaître une nouvelle perte visuelle après l'épisode initial27, le transfert du sang de la partie endommagée vers la partie saine de la $\mathrm{TNO}^{27}$ et la destruction initiale de la CFNR dans un disque optique encombré offrant plus d'espace pour les FNR survivantes ${ }^{28}$. Toutefois, ces idées hypothétiques ont été largement discréditées et la cause demeure non identifiée dans la plupart des épisodes de NOIA-NA récurrente ${ }^{6}$.

Dans la plupart des cas de NOIA-NA primaire, il est impossible d'établir une cause unique avec certitude. Malgré cela, il incombe aux fournisseurs de soins oculovisuels, de concert avec d'autres membres de l'équipe de soins de santé du patient, de détecter les facteurs de risque prédisposants et précipitants, dont certains peuvent être atténués. Ce faisant, la morbidité oculaire peut être réduite au minimum en diminuant le risque d'atteinte oculaire contralatérale et de récurrence ipsilatérale. De plus, une meilleure gestion des maladies systémiques sous-jacentes peut réduire le risque de mortalité systémique.

Dans ce cas-ci, l'objectif principal des soins après la NOIA-NA du patient était de discuter avec lui et de modifier tout facteur de risque systémique potentiel prédisposant ou précipitant. Toutefois, le traitement de la NOIA-NA au moyen de corticostéroïdes systémiques suscite une certaine controverse. Une vaste étude prospective portant sur 696 yeux a révélé que, dans les yeux présentant une acuité visuelle initiale de 20/70 ou plus faible, examinés dans les deux semaines suivant le début du traitement, l'acuité visuelle s'est améliorée chez 70 \% dans le groupe traité, contre $41 \%$ chez les patients du groupe non traité2 ${ }^{2}$. D'autres réfutent la validité de cette étude, citant plusieurs incohérences $^{30}$. Comme dans le présent cas l'acuité visuelle de l'œil droit au moment de la présentation ne répond pas aux critères de l'étude susmentionnée, les corticostéroïdes systémiques n’ont pas été pris en compte.

L'acuité visuelle et la perte du champ visuel sont des conséquences courantes de la NOIA-NA, mais ce ne sont pas les seuls symptômes avec lesquels les patients doivent composer à la suite de dommages ischémiques à la TNO. La perte de la sensibilité au contraste et la photophobie sont des problèmes débilitants qui résultent d'une NOIA-NA et qui peuvent avoir autant d'impact que la perte d'acuité visuelle. Le monde est composé de nuances infinies, et une faible sensibilité au contraste empêche les patients de déchiffrer ces changements subtils dans leur monde visuel. Les patients se plaignent souvent qu'il n'y a pas assez ou trop de lumière dans l'environnement, ce qui peut causer des problèmes de lueur ou de détection des contours des objets ${ }^{31}$. En outre, une association entre la sensibilité au contraste et l'indépendance fonctionnelle a été démontrée ${ }^{31,32}$. Il est donc important que les spécialistes de la réadaptation visuelle s'occupent des quatre principaux symptômes visuels après une NOIA-NA, soit l'acuité visuelle, le contraste, la photophobie et la perte du champ visuel. •

\section{RÉFÉRENCES}

1. Ischemic Optic Neuropathy Decompression Trial Research Group. Optic nerve decompression surgery for nonarteritic anterior ischemic optic neuropathy (NAION) is not effective and may be harmful. JAMA 1995;273:625-32.

2. Hayreh SS, Zimmerman MB. Nonarteritic anterior ischemic optic neuropathy: natural history of visual outcome. Ophthalmology 2008;115:298-305.

3. Hayreh SS. Management of ischemic optic neuropathies. Ind J Ophthalmol 2011;59:123-6.

4. Hayreh SS. Ischemic optic neuropathies-where are we now? Graefes Arch Clin Exp Ophthalmol 2013;251:1873-84.

5. Beri M, Klugman MR, Kohler JA, Hayreh SS. Anterior ischemic optic neuropathy. VII. Incidence of bilaterality and various influencing factors. Ophthalmology 1987;94:1020-8.

6. Hayreh SS, Podhajsky PA, Zimmerman B. Ipsilateral recurrence of non-arteritic anterior ischemic optic neuropathy. Am J Ophthalmol 2001;132:734-42.

7. Miller N, Subramanian P, Patel V. Walsh \& Hoyt's clinical neuroophthalmology: The essentials, vol. 3. Philadelphia, PA: Wolters Kluwer, 2016:145-160.

8. Hayreh SS. Ischemic optic neuropathy. Prog Retin Eye Res 2009 Jan;28(1):34-62.

9. Hayreh SS. Blood supply of the optic nerve head and its role in optic atrophy, glaucoma, and oedema of the optic disc. Br J Ophthalmol 1969;53:721-48.
10. Hayreh SS. The 1994 Von Sallman Lecture. The optic nerve head circulation in health and disease. Exp Eye Res 1995;61:259-72.

11. Wright Mayes E, Cole ED, Dang S, et al. Optical coherence tomography angiography in nonarteritic anterior ischemic optic neuropathy. J Neuroophthalmol 2017 Dec;37(4):358-64.

12. Liu CH, Kao LY, Sun MH, Wu WC, Chen HS. Retinal vessel density in optical coherence tomography angiography in optic atrophy after nonarteritic anterior ischemic optic neuropathy. J Ophthalmol 2017;2017:9632647.

13. Hayreh SS. Acute ischemic disorders of the optic nerve: pathogenesis, clinical manifestations and management. Ophthalmol Clin North Am 1996;9:407-42.

14. Hayreh SS. Blood flow in the optic nerve head and factors that may influence it. Prog Retin Eye Res 2001;20:595-624.

15. Hayreh SS, Zimmerman MB. Nonarteritic anterior ischemic optic neuropathy: clinical characteristics in diabetic patients versus nondiabetic patients. Ophthalmology 2008;115(10):1818-25.

16. Jacobson DM, Vierkant RA, Belongia EA. Nonarteritic anterior ischemic optic neuropathy. A case-control study of potential risk factors. Arch Ophthalmol 1997;115:1403-07.

17. Mojon DS, Hedges 3rd TR, Ehrenberg B, et al. Association between sleep apnea syndrome and nonarteritic anterior ischemic optic neuropathy. Arch Ophthalmol 2002;120:601-5.

18. Hayreh SS, Zimmerman MB. Nonarteritic anterior ischemic optic neuropathy: refractive error and its relationship to cup/disc ratio. 
Ophthalmology 2008;115: 2275-81.

19. Hayreh SS. Anterior ischemic optic neuropathy. IV. Occurrence after cataract extraction. Arch. Ophthalmol 1980;98:1410-6.

20. Hayreh SS. In vivo choroidal circulation and its watershed zones. Eye 1990;4:273-89.

21. Hayreh SS. Role of nocturnal arterial hypotension in the development of ocular manifestations of systemic arterial hypertension. Curr Opin Ophthalmol 1999;10(6):474-82.

22. Hayreh SS, Podhajsky P, Zimmerman MB. Role of nocturnal arterial hypotension in optic nerve head ischemic disorders. Ophthalmologica 1999;213:76-96.

23. Hayreh SS, Zimmerman MB, Podhajsky P, Alward WL. Nocturnal arterial hypotension and its role in optic nerve head and ocular ischemic disorders. Am J Ophthalmol 1994;117:603-24.

24. Hayreh SS, Podhajsky PA, Zimmerman B. Nonarteritic anterior ischemic optic neuropathy: time of onset of visual loss. Am J Ophthalmol 1997;124:641-7.

25. Newman NJ, Scherer R, Langenberg P, et al. The fellow eye in NAION: report from the ischemic optic neuropathy decompression trial follow-up study. Am J Ophthalmol 2008;134:317-28.

26. Hayreh SS, Zimmerman MB. Optic disc edema in non-arteritic anterior ischemic optic neuropathy. Graefes Arch Clin Exp Oph- thalmol 2007;245:1107-21.

27. Beck RW, Savino PJ, Schatz NJ, Smith CH, Sergott R. Anterior ischemic optic neuropathy: Recurrent episode in the same eye. Br J Ophthalmol 1983;67:705-9.

28. Hamed LM, Purvin V, Rosenberg M. Recurrent anterior ischemic optic neuropathy in young adults. J Clin Neuroophthalmol 1988 Dec;8(4):239-48

29. Hayreh SS, Zimmerman MB. Non-arteritic anterior ischemic optic neuropathy: Role of systemic corticosteroid therapy. Graefes Arch Clin Exp Ophthalmol 2008;246:1029-46.

30. Atkins EJ, Bruce BB, Newman NJ, Biousse V. Treatment of nonarteritic anterior ischemic optic neuropathy. Surv Ophthalmol 2010;55(1):47-63.

31. Dos Santos NA, Andrade SM. Visual contrast sensitivity in patient with impairment of functional independence after stroke. BMC Neurol 2012 Sep 12;12:90.

32. Wolter M, Preda S. Visual deficits following stroke: maximizing participation in rehabilitation. Top Stroke Rehabil 2006 Summer;13(3):12-21. 